\title{
The role of the computational variables on the performances of COSMO- SAC model: a combined theoretical and experimental investigation.
}

\author{
Gabrielly Miyazaki1 ${ }^{1,2}$, Bernardino Tirri² ${ }^{2}$ Olivier Baudouin ${ }^{3}$, Alain Valtz ${ }^{1}$, Céline Houriez ${ }^{1}$, Christophe Coquelet ${ }^{1 *}$ \\ and Carlo Adamo $1,2,4 *$ \\ 1) Mines ParisTech, PSL University, CTP - Centre of Thermodynamics of Processes, 35 Rue Saint Honoré, \\ 77305 Fontainebleau, France; \\ 2) Chimie ParisTech, PSL University, i-CLeHS - Institute of Chemistry for Life and Health Science, 11, Rue \\ Pierre et Marie Curie, 75015 Paris, France \\ 3) ProSim SA - Software \& Services in Process Simulation, Immeuble Stratège A, 51 Rue Ampère, F-31670 \\ LABEGE, France \\ 4) Institut Universitaire de France, 103 Boulevard Saint Michel, F-75005 Paris, France.
}

\section{SUPPORTING INFORMATION}


Table S1. Specifications and suppliers of chemicals used.

\begin{tabular}{|c|c|c|c|c|c|c|}
\hline \multirow[b]{2}{*}{$\begin{array}{l}\text { Chemical } \\
\text { name }\end{array}$} & \multirow[b]{2}{*}{ CAS } & \multirow{2}{*}{$\begin{array}{l}\text { Molar } \\
\text { Mass } \\
(\mathrm{g} / \mathrm{mol})\end{array}$} & \multirow[b]{2}{*}{$\begin{array}{l}\text { Purity } \\
(\% w t)\end{array}$} & \multirow[b]{2}{*}{ Suplier } & \multicolumn{2}{|c|}{ Refractive index } \\
\hline & & & & & $\begin{array}{c}\text { Measured a }^{\mathrm{a}} \text { at } \\
298.15 \mathrm{~K} \text { and } \\
101.325 \mathrm{kPa}\end{array}$ & literature \\
\hline $\begin{array}{l}\text { Furan } \\
\text { (stabilized) }\end{array}$ & $110-00-9$ & 68.08 & $\geq 99.0$ & $\begin{array}{c}\text { SIGMA } \\
\text { ALDRICH }\end{array}$ & 1.42166 & 1.41781 \\
\hline Cyclohexane & $110-82-7$ & 84.16 & $\geq 99.0$ & $\begin{array}{c}\text { SIGMA } \\
\text { ALDRICH }\end{array}$ & 1.42633 & 1.42354 \\
\hline
\end{tabular}

${ }^{a}$ Apparatus: Anton Paar ABBEMAT. Standard uncertainties $u$ are refractive index $u(n D)=0.0001$, temperature $u(T)=0.02 \mathrm{~K}$ and pressure $\mathrm{u}(\mathrm{P})=0.029 \mathrm{kPa}$,

${ }^{\mathrm{b}}$ Values from DIPPR database. ${ }^{44}$ 
Table S2. DIPPR Coefficients for pure components vapor pressure in Pa (Eq. 3).

\begin{tabular}{lccccc}
\hline & $\mathrm{A}$ & $\mathrm{B}$ & $\mathrm{C}$ & $\mathrm{D}$ & $\mathrm{E}$ \\
\hline furan $^{\mathrm{a}}$ & 74.738 & -5417 & -8.0636 & $7.47 \mathrm{E}-06$ & 2 \\
cyclohexane $^{\mathrm{b}}$ & 51.087 & -5226.4 & -4.2278 & $9.76 \mathrm{E}-18$ & 6 \\
\hline
\end{tabular}

${ }^{a}$ Uncertainty $<1 \%$ (compared with experimental data from Zhang et.al ${ }^{2 x}$ ). Coefficients from DIPPR database. ${ }^{44}$

${ }^{\mathrm{b}}$ Uncertainty $<1 \%$ (compared with experimental data from Kobe et.al ${ }^{3 \mathrm{x}}$ ). Coefficients from DIPPR database. ${ }^{44}$ 
Table S3. Atomic radii $(\AA)$ used in the present work. For comparison purposes are also reported the vdW raddi as defined by Bondi ${ }^{53}$.

\begin{tabular}{cccc}
\hline Element & vdW & Klamt & UFF \\
\hline $\mathrm{H}$ & 1.20 & 1.30 & 1.443 \\
$\mathrm{C}$ & 1.70 & 2.00 & 1.9255 \\
$\mathrm{O}$ & 1.52 & 1.72 & 1.75 \\
\hline
\end{tabular}


Table S4. Values of the parameters used in the COSMO-SAC model, as implemented in references 33 and 35.

\begin{tabular}{|c|c|}
\hline \multicolumn{2}{|c|}{ Universal Parameters } \\
\hline Parameter & Value \\
\hline$a_{e f f}\left(\AA^{2}\right)$ & 7.25 \\
\hline$f_{\text {decay }}$ & 3.57 \\
\hline$\sigma_{0}\left(\mathrm{e} / \AA^{2}\right)$ & 0.007 \\
\hline$r\left(\AA^{3}\right)$ & 66.69 \\
\hline$q\left(\AA^{2}\right)$ & 79.53 \\
\hline $\mathrm{z}$ & 10 \\
\hline $\mathrm{A}_{\mathrm{ES}}\left(\mathrm{kcal} \cdot \mathrm{mol}^{-1} \cdot \AA^{4} \cdot \mathrm{e}^{-2}\right)$ & 6525.69 \\
\hline $\mathrm{B}_{\mathrm{ES}}\left(\mathrm{kcal} \cdot \mathrm{mol}^{-1} \cdot \AA^{4} \cdot \mathrm{e}^{-2} \cdot \mathrm{K}^{2}\right)$ & $1.4859 \times 10^{8}$ \\
\hline $\mathrm{c}_{\mathrm{OH}-\mathrm{OH}}\left(\mathrm{kcal}_{\mathrm{mol}} \mathrm{mo}^{-1} \cdot \AA^{4} \cdot \mathrm{e}^{-2}\right)$ & 4013.78 \\
\hline $\mathrm{c}_{O T-O T}\left(\mathrm{kcal} \cdot \mathrm{mol}^{-1} \cdot \AA^{4} \cdot \mathrm{e}^{-2}\right)$ & 932.31 \\
\hline $\mathrm{c}_{O H-O T}\left(\mathrm{kcal} \cdot \mathrm{mol}^{-1} \cdot \AA^{4} \cdot \mathrm{e}^{-2}\right)$ & 3016.43 \\
\hline \multicolumn{2}{|c|}{ Dispersive parameters } \\
\hline$\omega$ & 0.27027 or -0.27027 \\
\hline \multicolumn{2}{|c|}{ Dispersion parameters for atom types } \\
\hline Atom type (hybridization type) & $\left(\varepsilon_{\text {atom }, i} / k_{B}\right) / \mathrm{K}$ \\
\hline $\mathrm{C}\left(\mathrm{sp}^{3}\right)$ & 115.7023 \\
\hline $\mathrm{C}\left(\mathrm{sp}^{2}\right)$ & 117.4650 \\
\hline $\mathrm{C}(\mathrm{sp})$ & 66.0691 \\
\hline$-\mathrm{O}-$ & 95.6184 \\
\hline $\mathrm{H}(\mathrm{OH})(\mathrm{H}-\mathrm{O}$ bond not in water $)$ & 19.3477 \\
\hline
\end{tabular}


Table S5. Experimental apparent Henry's law constant (H, MPa) and IDAC $\left(\boldsymbol{\gamma}^{\infty}\right)$ at different temperatures and atmospheric pressure $(\mathrm{u}(\mathrm{T})=0.02 \mathrm{~K}, \mathrm{u}(\mathrm{P})=0.1 \mathrm{kPa}$ of furan in cyclohexane at temperatures from 283 to $318 \mathrm{~K}$.

\begin{tabular}{cccc}
\hline $\mathrm{T}$ & $\mathrm{H}$ & $\boldsymbol{\gamma}^{\infty}$ & $\mathrm{u}\left(\mathrm{H}_{\mathrm{i}}\right) / \mathrm{H}_{\mathrm{i}}$ \\
\hline 283.6 & 0.101 & 2.28 & $4.4 \%$ \\
288.6 & 0.123 & 2.26 & $0.67 \%$ \\
293.5 & 0.138 & 2.07 & $2.1 \%$ \\
298.2 & 0.162 & 2.02 & $1.6 \%$ \\
303.4 & 0.192 & 1.97 & $4.7 \%$ \\
308.5 & 0.224 & 1.92 & $1.8 \%$ \\
313.4 & 0.262 & 1.83 & $2.7 \%$ \\
318.4 & 0.289 & 1.76 & $2.0 \%$ \\
\hline
\end{tabular}


Table S6. Combinatorial (comb), residual (res) and dispersion (dsp) contributions to the IDACs of furan in tolouene, computed with PBE0, M06X and B3LYP functionals. Data from the VT2005 database are also reported.

\begin{tabular}{|c|c|c|c|c|c|c|c|c|}
\hline $\begin{array}{c}\mathrm{T} \\
(\mathrm{K}) \\
\end{array}$ & $\ln \gamma_{\mathrm{i}}^{\text {calc }}$ & $\begin{array}{c}\text { PBE0 } \\
\ln \gamma_{\mathrm{i}}{ }^{\mathrm{comb}}\end{array}$ & $\ln \gamma_{\mathrm{i}}{ }^{\text {res }}$ & $\ln \gamma_{\mathrm{i}}^{\mathrm{dps}}$ & $\ln \gamma_{\mathrm{i}}^{\text {calc }}$ & $\begin{array}{l}\text { M06-2X } \\
\ln \gamma_{\mathrm{i}}{ }^{\text {comb }}\end{array}$ & $\ln \gamma_{\mathrm{i}}{ }^{\text {res }}$ & $\ln \gamma_{\mathrm{i}}^{\mathrm{dps}}$ \\
\hline 293.15 & 0.0296 & -0.1505 & 0.1750 & 0.0051 & 0.0383 & -0.1507 & 0.1839 & 0.0051 \\
\hline 298.15 & 0.0286 & -0.1505 & 0.1740 & 0.0051 & 0.0363 & -0.1507 & 0.1820 & 0.0051 \\
\hline 303.16 & 0.0266 & -0.1505 & 0.1721 & 0.0051 & 0.0354 & -0.1507 & 0.1810 & 0.0051 \\
\hline 308.19 & 0.0257 & -0.1505 & 0.1711 & 0.0051 & 0.0334 & -0.1507 & 0.1791 & 0.0051 \\
\hline 313.17 & 0.0247 & -0.1505 & 0.1701 & 0.0051 & 0.0325 & -0.1507 & 0.1781 & 0.0051 \\
\hline 318.12 & 0.0227 & -0.1505 & 0.1682 & 0.0051 & 0.0305 & -0.1507 & 0.1762 & 0.0051 \\
\hline 323.09 & 0.0218 & -0.1505 & 0.1672 & 0.0051 & 0.0296 & -0.1507 & 0.1752 & 0.0051 \\
\hline $\mathrm{T}$ & & B3LYP & & & & VT-2005 & & \\
\hline$(\mathrm{K})$ & $\ln \gamma_{\mathrm{i}}^{\text {calc }}$ & $\ln \gamma_{\mathrm{i}}{ }^{\mathrm{comb}}$ & $\ln \gamma_{\mathrm{i}}{ }^{\text {res }}$ & $\ln \gamma_{\mathrm{i}}^{\mathrm{dps}}$ & $\ln \gamma_{\mathrm{i}}^{\text {calc }}$ & $\ln \gamma_{\mathrm{i}}{ }^{\mathrm{comb}}$ & $\ln \gamma_{\mathrm{i}}^{\mathrm{res}}$ & $\ln \gamma_{\mathrm{i}}^{\mathrm{dps}}$ \\
\hline 293.15 & 0.0751 & -0.1509 & 0.2210 & 0.0051 & 0.09167 & -0.189743 & 0.2763 & 0.0051 \\
\hline 298.15 & 0.0723 & -0.1509 & 0.2182 & 0.0051 & 0.08893 & -0.189743 & 0.2736 & 0.0051 \\
\hline 303.16 & 0.0695 & -0.1509 & 0.2154 & 0.0051 & 0.08618 & -0.189743 & 0.2709 & 0.0051 \\
\hline 308.19 & 0.0677 & -0.1509 & 0.2135 & 0.0051 & 0.08434 & -0.189743 & 0.2690 & 0.0051 \\
\hline 313.17 & 0.0649 & -0.1509 & 0.2107 & 0.0051 & 0.08158 & -0.189743 & 0.2663 & 0.0051 \\
\hline 318.12 & 0.0620 & -0.1509 & 0.2079 & 0.0051 & 0.07973 & -0.189743 & 0.2644 & 0.0051 \\
\hline 323.09 & 0.0602 & -0.1509 & 0.2060 & 0.0051 & 0.07696 & -0.189743 & 0.2616 & 0.0051 \\
\hline
\end{tabular}


Table S7. Percent Mean Absolute Deviations (\% MAD) of IDAC of furan for different basis set and functionals

\begin{tabular}{cccccc}
\hline solvent & UNIFAC & VT 2005 & Basis set & M062X & PBE0 \\
\hline \multirow{2}{*}{ toluene } & \multirow{2}{*}{$13.9 \%$} & \multirow{2}{*}{$5.7 \%$} & $6-31++\mathrm{G}(2 \mathrm{df}, \mathrm{p})$ & 4.0 & 2.5 \\
& & & $6-311 \mathrm{G}(2 \mathrm{df}, \mathrm{p})$ & 0.6 & 0.5 \\
& & & $6-311++\mathrm{G}(2 \mathrm{df}, \mathrm{p})$ & 2.7 & 1.7 \\
\hline \multirow{3}{*}{ cyclohexane } & \multirow{2}{*}{$77.6 \%$} & \multirow{2}{*}{$60.5 \%$} & $6-31++\mathrm{G}(2 \mathrm{df}, \mathrm{p})$ & 44.5 & 36.5 \\
& & & $6-311+\mathrm{G}(2 \mathrm{df}, \mathrm{p})$ & 40.2 & 29.7 \\
& & & &
\end{tabular}


Table S8. IDAC of Furan in Toluene computed using the PBE0 functionals and different basis sets.

\begin{tabular}{ccccccccc}
\hline $6-31 \mathrm{G}(\mathrm{d})$ & $6-311 \mathrm{G}(\mathrm{d})$ & $6-31 \mathrm{G}(\mathrm{d}, \mathrm{p})$ & $6-31 \mathrm{G}(2 \mathrm{df}, \mathrm{p})$ & $6-311++\mathrm{G}(\mathrm{d})$ & $6-31++\mathrm{G}(\mathrm{d}, \mathrm{p})$ & $6-31++\mathrm{G}(2 \mathrm{df}, \mathrm{p})$ & $6-311 \mathrm{G}(2 \mathrm{df}, \mathrm{p})$ & $6-311++\mathrm{G}(2 \mathrm{df}, \mathrm{p})$ \\
\hline 1.046 & 1.029 & 1.046 & 1.038 & 1.105 & 1.072 & 1.062 & 1.030 & 1.052 \\
1.045 & 1.028 & 1.044 & 1.036 & 1.102 & 1.070 & 1.059 & 1.029 & 1.050 \\
1.043 & 1.026 & 1.043 & 1.034 & 1.098 & 1.068 & 1.057 & 1.027 & 1.048 \\
1.041 & 1.025 & 1.041 & 1.032 & 1.095 & 1.065 & 1.055 & 1.026 & 1.046 \\
1.039 & 1.024 & 1.039 & 1.031 & 1.092 & 1.063 & 1.053 & 1.025 & 1.045 \\
1.037 & 1.023 & 1.037 & 1.029 & 1.090 & 1.061 & 1.051 & 1.023 & 1.043 \\
1.036 & 1.022 & 1.036 & 1.028 & 1.087 & 1.059 & 1.049 & 1.022 & 1.041 \\
\hline
\end{tabular}


Table S9. IDAC of Furan in Cyclohexane computed using different cavity radii and the using PBE0/6-311G(2df,p) level of theory

\begin{tabular}{cc}
\hline Klamt & UFF Radii \\
\hline 2.894 & 2.173 \\
2.801 & 2.117 \\
2.715 & 2.064 \\
2.640 & 2.019 \\
2.562 & 1.971 \\
2.490 & 1.927 \\
2.426 & 1.888 \\
\hline
\end{tabular}


Table S10 IDAC of Furan in $n$-Alkanes.

\begin{tabular}{|c|c|c|c|c|c|c|}
\hline \multirow{2}{*}{ n-alkane } & \multirow{2}{*}{$\mathrm{T}(\mathrm{K})$} & \multirow{2}{*}{$\exp$} & \multirow{2}{*}{ VT 2005} & \multirow[t]{2}{*}{ UNIFAC } & \multicolumn{2}{|c|}{ PBE0/6-311G(2df.p) } \\
\hline & & & & & Klamt & UFF Radii \\
\hline \multirow{5}{*}{ C5 } & 283.40 & 1.570 & 3.358 & 3.329 & 2.719 & 2.104 \\
\hline & 288.30 & 1.450 & 3.241 & 3.287 & 2.639 & 2.052 \\
\hline & 293.20 & 1.290 & 3.133 & 3.248 & 2.564 & 2.005 \\
\hline & 298.10 & 1.050 & 3.033 & 3.211 & 2.494 & 1.960 \\
\hline & 302.10 & 0.740 & 2.956 & 3.182 & 2.440 & 1.925 \\
\hline \multirow{7}{*}{ C6 } & 293.40 & 1.760 & 3.020 & 2.768 & 2.463 & 1.897 \\
\hline & 298.20 & 1.760 & 2.925 & 2.737 & 2.396 & 1.856 \\
\hline & 303.20 & 1.700 & 2.832 & 2.707 & 2.332 & 1.815 \\
\hline & 308.20 & 1.680 & 2.746 & 2.678 & 2.271 & 1.777 \\
\hline & 313.00 & 1.620 & 2.669 & 2.652 & 2.217 & 1.743 \\
\hline & 318.00 & 1.470 & 2.593 & 2.625 & 2.164 & 1.709 \\
\hline & 323.10 & 1.320 & 2.522 & 2.601 & 2.113 & 1.677 \\
\hline \multirow{7}{*}{ C7 } & 293.30 & 1.570 & 2.897 & 2.445 & 2.342 & 1.792 \\
\hline & 298.20 & 1.670 & 2.803 & 2.417 & 2.277 & 1.752 \\
\hline & 303.10 & 1.590 & 2.715 & 2.391 & 2.217 & 1.714 \\
\hline & 308.30 & 1.570 & 2.629 & 2.364 & 2.157 & 1.677 \\
\hline & 313.20 & 1.530 & 2.553 & 2.341 & 2.104 & 1.644 \\
\hline & 318.10 & 1.520 & 2.482 & 2.318 & 2.054 & 1.613 \\
\hline & 323.10 & 1.490 & 2.414 & 2.297 & 2.007 & 1.583 \\
\hline \multirow{5}{*}{ C8 } & 293.40 & 1.570 & 2.767 & 2.207 & 2.225 & 1.691 \\
\hline & 298.30 & 1.560 & 2.677 & 2.182 & 2.163 & 1.653 \\
\hline & 303.40 & 1.530 & 2.589 & 2.158 & 2.103 & 1.616 \\
\hline & 308.30 & 1.500 & 2.511 & 2.135 & 2.049 & 1.582 \\
\hline & 323.10 & 1.470 & 2.305 & 2.074 & 1.905 & 1.493 \\
\hline \multirow{7}{*}{ C9 } & 293.40 & 1.610 & 2.639 & 2.024 & 2.110 & 1.597 \\
\hline & 298.30 & 1.540 & 2.553 & 2.001 & 2.051 & 1.561 \\
\hline & 303.20 & 1.530 & 2.472 & 1.979 & 1.996 & 1.527 \\
\hline & 308.20 & 1.450 & 2.396 & 1.958 & 1.943 & 1.495 \\
\hline & 313.20 & 1.430 & 2.324 & 1.938 & 1.894 & 1.465 \\
\hline & 318.20 & 1.400 & 2.258 & 1.919 & 1.848 & 1.436 \\
\hline & 323.10 & 1.350 & 2.197 & 1.901 & 1.806 & 1.410 \\
\hline \multirow{7}{*}{ C10 } & 293.20 & 1.430 & 2.519 & 1.877 & 2.006 & 1.512 \\
\hline & 298.10 & 1.400 & 2.436 & 1.855 & 1.950 & 1.478 \\
\hline & 303.20 & 1.340 & 2.356 & 1.834 & 1.895 & 1.445 \\
\hline & 308.20 & 1.300 & 2.282 & 1.815 & 1.845 & 1.414 \\
\hline & 313.20 & 1.240 & 2.214 & 1.796 & 1.799 & 1.385 \\
\hline & 318.10 & 1.230 & 2.152 & 1.779 & 1.756 & 1.359 \\
\hline & 323.20 & 1.200 & 2.091 & 1.762 & 1.714 & 1.333 \\
\hline
\end{tabular}




\begin{tabular}{|c|c|c|c|c|c|c|}
\hline \multirow{7}{*}{ C12 } & 293.20 & 1.430 & 2.301 & 1.651 & 1.817 & 1.361 \\
\hline & 298.10 & 1.400 & 2.225 & 1.632 & 1.766 & 1.330 \\
\hline & 303.20 & 1.340 & 2.151 & 1.614 & 1.716 & 1.300 \\
\hline & 308.20 & 1.300 & 2.084 & 1.597 & 1.671 & 1.273 \\
\hline & 313.10 & 1.240 & 2.022 & 1.580 & 1.629 & 1.247 \\
\hline & 318.00 & 1.230 & 1.965 & 1.565 & 1.590 & 1.223 \\
\hline & 323.10 & 1.200 & 1.909 & 1.550 & 1.552 & 1.200 \\
\hline \multirow{7}{*}{ C16 } & 293.20 & 1.300 & 1.946 & 1.355 & 1.519 & 1.129 \\
\hline & 298.20 & 1.270 & 1.879 & 1.339 & 1.475 & 1.103 \\
\hline & 303.30 & 1.210 & 1.816 & 1.324 & 1.433 & 1.077 \\
\hline & 308.20 & 1.190 & 1.760 & 1.310 & 1.395 & 1.055 \\
\hline & 313.10 & 1.170 & 1.707 & 1.297 & 1.360 & 1.034 \\
\hline & 318.10 & 1.160 & 1.657 & 1.284 & 1.327 & 1.013 \\
\hline & 323.10 & 1.170 & 1.611 & 1.272 & 1.295 & 0.994 \\
\hline
\end{tabular}


Figure S1. $\sigma$-profiles for n-hexane and n-dodecane, computed using the PBE0 and M06-2X functionals. Data from the VT-2005 set are also reported.

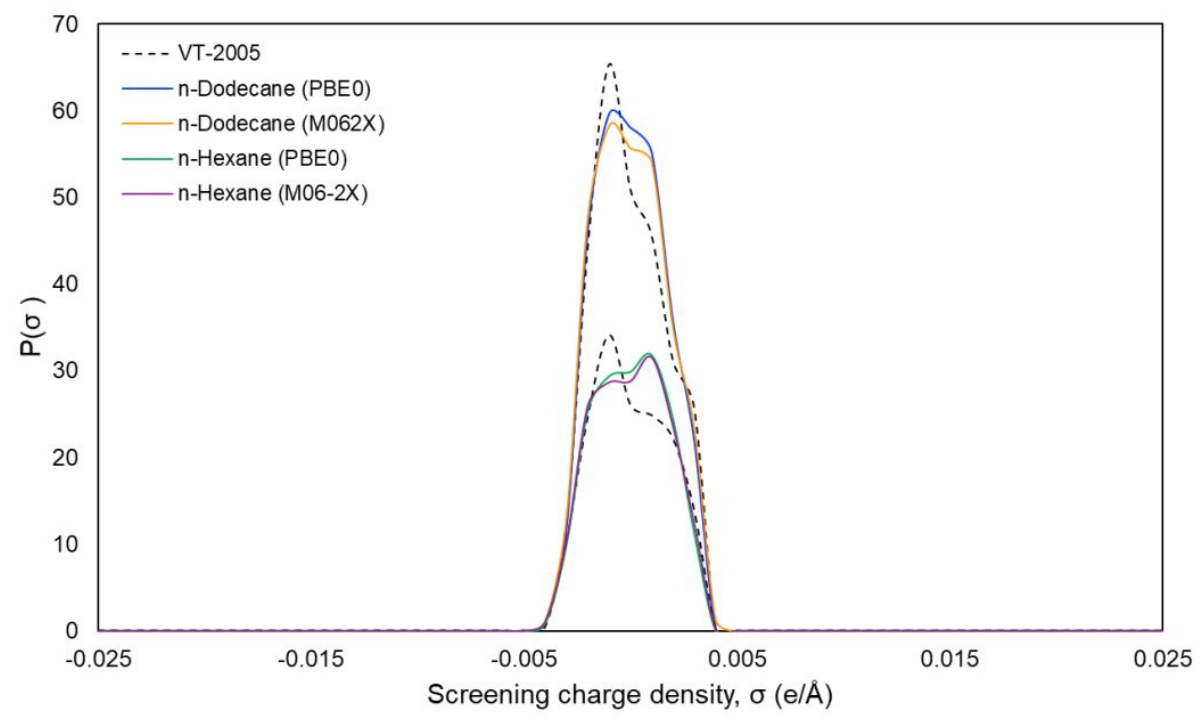


Figure S2. Conformational isomers of $n$-pentane, their IDACs and $\sigma$-profile, computed at the PBE0 level.
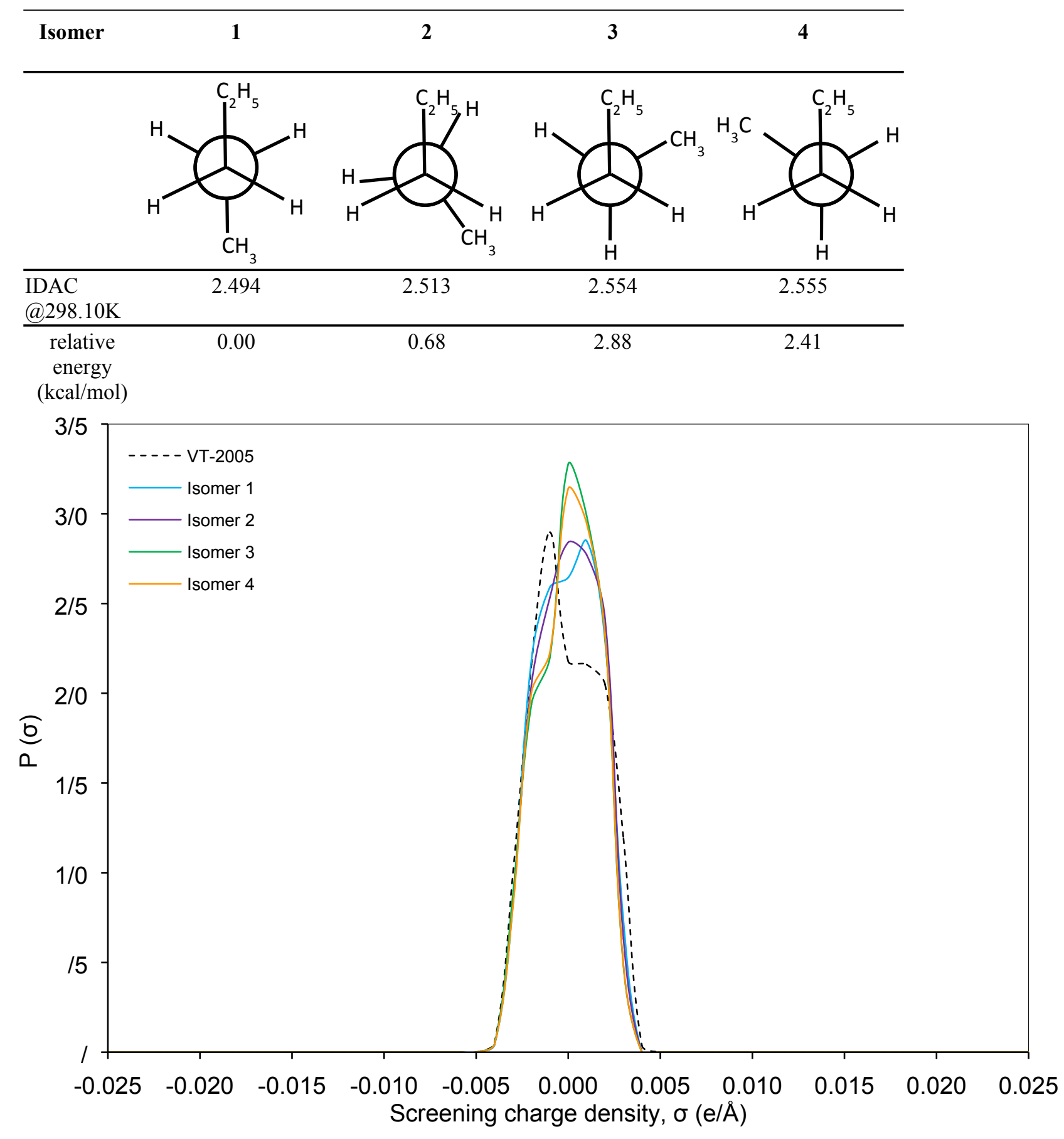
Details on the calculations of $\sigma$-profile from Gaussian COSMO-RS file, as implemented in the Simulis Thermodynamics software.

The screening charge from the QM/COSMO calculation are averaged by the following expression:

$$
\sigma_{m}=\frac{\sum_{n} \sigma_{n}{ }^{*} \frac{r_{n}^{2} r_{e f f}^{2}}{r_{n}^{2}+r_{e f f}^{2}} \exp \left(-f_{\text {decay }} \frac{d_{m n}^{2}}{r_{n}^{2}+r_{e f f}^{2}}\right)}{\sum_{n_{r_{n}}^{2}+r_{e f f}^{2}} \exp \left(-f_{\text {decay }} \frac{d_{m n}^{2} r_{r_{n}^{2}+r_{e f f}^{2}}^{2}}{2}\right)}
$$

Where $\sigma_{n}{ }^{*}$ is the original surface charge of the $n$th segment and $r_{n}$ is radius of the segment $n$, both taken directly from the COSMORS file. The $d_{m n}$ is the distance (in $\AA$ ) between the centers of the surface segments $n$ and $m$ calculate by eq (2). All the parameters used to the sigma-profile construction are defined in the Table S1.

$d_{m n}=\sqrt{\left(x_{m}-x_{n}\right)^{2}+\left(y_{m}-y_{n}\right)^{2}+\left(z_{m}-z_{n}\right)^{2}}$

Table S1. Parameters used in the sigma-profile calculation.

\begin{tabular}{cccc}
\hline Parameters & & & \\
\hline$a_{\text {eff }}$ & $7.5 \AA^{2}$ & effective area for averaging \\
$r_{\text {eff }}$ & $=\sqrt{a_{e f f} / \pi}$ & effective radius for averaging \\
$f_{\text {decay }}$ & 3.57 & empiric parameter \\
\hline
\end{tabular}

